\title{
The Relationship Between Stress and Coping in Table Tennis
}

\author{
by \\ Dora Kurimay ${ }^{1}$, Alison Pope-Rhodius ${ }^{2}$, Miran Kondric ${ }^{3}$
}

The aim of this study was to examine the relationship between cognitive competitive anxiety intensity and coping strategies in table tennis players. One hundred and two (102) US competitive table tennis players of age range from 10 to 60 filled out a Revised Competitive State Anxiety Inventory-2 (CSAI-2R, Cox et al., 2003) at least 30 minutes before the start of their tournament match and a Modified Cope questionnaire (MCOPE; Crocker and Graham, 1995) 15 minutes after they finished their match. Our study found significant differences between low and high cognitive competitive anxiety groups with regard to the use of coping strategies. The high cognitive competitive anxiety intensity group used significantly more behavioral disengagement (avoidance coping, $p \leq 0.05$ ), denial coping strategies (emotion focused coping, $p \leq 0.01$ ) compared to the low cognitive anxiety intensity group. Our results suggest that there is some connection between anxiety intensity and coping strategies. If the cognitive anxiety intensity (for example, intensity from worrying) is very high, an athlete might be more likely to use avoidance coping (such as behavioral disengagement) and emotion-focused coping (such as denial and venting of emotions) compared to athletes who have low cognitive competitive anxiety. Furthermore, gender differences in cognitive anxiety and direction were found. Confidence management techniques such as positive self-talk, breathing techniques and visualization should be taught to athletes to assist them in coping with their competitive anxiety better and to enhance their performance.

Key words: avoidance, competitive cognitive anxiety, coping strategies, emotion focused coping strategies, mental toughness, table tennis.

\section{Introduction}

Competitive anxiety and stress management are part of any kind of competitions. Competitive anxiety is a mental state which includes cognitive, somatic and emotional components (Gillham et al., 2014; Jones, 1995; Mellalieu et al., 2009; Woodman and Hardy, 2001). All these components affect how athletes handle stress (Mellalieu et al., 2009; Patel et al., 2010).

There are many different factors such as age, gender, life experience, skill level (Gan et al., 2009; Radochonski et al., 2011), control of the situation (Jones, 1995) and self-confidence (Gillham et al., 2014; Jones, 1995; Mellalieu et al.,

2009; Wagstaff et al., 2011), which have an impact on competitive anxiety. Using different coping strategies can also influence the effects of stress symptoms (for example worry and heart rate). Stress can even be interpreted as facilitative and can play an important role in performing well in competitive situations (Gillham et al., 2014; Mellalieu et al., 2009; Neil et al., 2006).

Sports-related coping research has found that problem-focused strategies are associated with positive effects, while emotion-focused strategies and avoidance coping strategies are associated with negative effects (Crocker and Graham, 1995; Kashdan et al., 2006). However,

1 - www.dorakurimay.com

2 - John F. Kennedy University, Sport Psychology Program, USA.

3 - University of Ljubljana, Faculty of Sport, Slovenia. 
Krohne and Hindel (1988) found that elite German table tennis players who used cognitive avoidance coping strategies during a match were more successful (they won more matches) compared to players who did not employ these strategies. In addition, avoidance coping was associated with less state anxiety after making performance errors compared to the use of problem-focused coping (Krohne and Hindel, 1988). Problem-focused coping strategies are targeted toward stressful situations and resolution of the problem, while avoidance coping protects against interfering thoughts and actions. Nevertheless, Roth and Cohen (1986) found that avoidance coping was better than problemfocused coping if the situation was uncontrollable, whereas problem-focused coping was better when there was the potential for control. Hence, in the Krohne and Hindel's study (1988), elite German table tennis players' avoidance coping was associated with reduced state anxiety and better performance as the situation was uncontrollable and there was a low level of situational control. Supporting this fact, Kaisidis-Rodafinos et al. (1997) suggested that under certain circumstances such as low situational control, avoidance coping might be more effective in reducing stress than problem-focused coping. Another study by Anshel and Si (2008) also supported the idea that copying style was a function of the type of a stressful event, where "stressful event" was defined as when an athlete felt worry or anxiety about an upcoming event, thus their focus was only on cognitive symptoms. Moreover, Anshel and Si (2008) found that avoidance coping could be more common than problem-focused coping in certain stressful events in elite Chinese athletes.

$$
\text { Hatzigeorgiadis and Chroni (2007) }
$$

studied Greek elite male swimmers' precompetitive anxiety and coping strategies during competition. Specifically, the participants of the study completed a short test of anxiety intensity and direction before their competition and a coping questionnaire after their event. According to the findings, the intensity of cognitive anxiety had low to moderate correlation with a coping approach (problem-focused strategies, however, only planning was significant) and a low to moderate positive relationship with avoidance coping strategies (none of which were statistically significant).
In another study, Ntoumanes and Biddle (2000) examined how coping strategies related to the level of intensity and direction (debilitative or facilitative) of anxiety of sports. British university athletes had to recall a recent stressful situation in their sport, the coping strategies that they used and the intensity and direction of their anxiety symptoms. The perception of facilitative cognitive anxiety (facilitative direction of anxiety) was related to problem-focused coping, while high levels of cognitive intensity were related to emotion focused coping and avoidance.

Based on the Ntoumanis and Biddle's study (2000), our assumption was that table tennis players with high cognitive competitive anxiety intensity would report greater use of emotionfocused and avoidance coping strategies compared to those with low cognitive competitive anxiety intensity. Furthermore, gender differences were expected between the level of anxiety and the preferred coping style.

\section{Material and Methods}

\section{Participants}

One hundred and two (102) competitive table tennis players aged 10 to 60 (80 males and 20 females) from the United States participated in the study. The participants' table tennis level was mainly intermediate based on the American ranking system. Intermediate players' rankings were between 1000 and 1700 and advanced players were between 1700 and 2200 .

\section{Measures}

The relationship of competitive anxiety and coping strategies in sport was studied based on a quantitative method of research using the SPSS (Statistical Package for the Social Sciences) program for statistical analysis. The study examined how independent variables (competitive cognitive anxiety intensity factors) influenced dependent variables (coping strategies). Furthermore, gender differences in the different use of coping strategies as well as the intensity and direction of competitive anxiety were investigated.

\section{Procedures}

The athletes were verbally invited to participate at table tennis tournaments in the USA and were asked to complete a demographic information sheet and the Revised Competitive State Anxiety Inventory-2 (CSAI-2-R; Cox et al., 
2003) a minimum of 30 and maximum of $90 \mathrm{~min}$ before the start of the competitive event. The timing of the inventory administration might have had an effect on the athletes' game, although the researchers did not receive any negative feedback, comments or complaints from the participants. Additionally, participants were asked to complete the Modified Cope questionnaire (MCOPE; Crocker and Graham, 1995) 15 min after their match.

The participants were protected following the ethical standards taken from the APA Code of Conducts (2003). Informed consent documents emphasizing confidentiality were given to each participant and discussed in detail prior to the questionnaires. Assent information was included to participants that were minors. Furthermore, a consent form was given to participants who were over age 18 and to the minors' parents/guardians. The researchers verified that the participants understood the documents and the research procedures.

\section{Statistical Analysis}

One-way ANOVA was used to examine how independent variables (competitive cognitive anxiety intensity factors) influenced dependent variables (coping strategies). Competitive anxiety intensity factors (high/low intensity) were the dependent variables and the 12 coping strategies were dependent variables. The 12 coping strategies were active coping, seeking social support for instrumental reasons, planning, increasing effort, suppression of competitive activities (these are problem-focused strategies), seeking social support for emotional reasons, denial, humor, venting of emotion, self-blame, wishful thinking (they are known as emotionfocused strategies) and behavioural disengagement (it is classified as avoidance coping).

Using the median of the competitive cognitive anxiety intensity scores, two grouping variables created for each ANOVA (high/low intensity). Participants who had exactly the same score as the median were taken out from the sample (i.e. 13 athletes). The cognitive anxiety intensity median was 20.00 . Therefore, those who had lower intensity scores than the median were put into a low intensity category $(\mathrm{N}=48)$, while those who had higher scores than the median were placed into a high intensity category $(\mathrm{N}=54)$.
A t-test was used to examine gender differences in the different use of coping strategies as well as the intensity and direction of competitive anxiety.

\section{Results}

The high cognitive anxiety intensity group used significantly more $(p \leq 0.05)$ avoidance coping (behavioural disengagement) compared to the low cognitive anxiety intensity group. Moreover, athletes in the high cognitive competitive anxiety intensity group used significantly more $(p \leq 0.01)$ denial coping strategies (emotion focused coping) compared to athletes who were in the low cognitive anxiety group (Table 1 and Figure 1).

Results from the study showed that gender differences did exist in cognitive anxiety intensity and direction. Women rated their cognitive anxiety higher than their male counterparts (Table 2). However, researchers could not verify any significant gender differences in copying style.

\section{Discussion}

The present study examined the relationships between cognitive competitive anxiety intensity and coping strategies in table tennis players. Our study found significant difference between low and high cognitive competitive anxiety groups with regard to the use of coping strategies.

A significantly higher use of avoidance coping in the high cognitive anxiety group compared to low cognitive competitive anxiety group is congruent with the results of the Hatzigeorgiadis and Chroni's study (2007). They also found low to moderate positive correlations between avoidance coping and the intensity of cognitive anxiety in competitive male swimmers even if their result was not statistically significant. Furthermore, Hatzigeorgiadis and Chroni (2007) examined only approach coping (problemfocused coping) along with avoidance coping and their study did not include emotion-focused coping strategies. Additionally, only male swimmers were surveyed in their research and their sample size was limited. The use of the Mental Readiness Form to assess pre-competition anxiety (MRF-3, Thomas et al., 1999) considering the validity of a single instrument is also questionable. Hence, although our result is 
congruent with the one reported in the Hatzigeorgiadis and Chroni's study (2007), our study highlights the possibility of some relationship between avoidance coping and a high level of cognitive anxiety even if we cannot make further generalizations based on this result.

In certain conditions, avoidance coping can be effective in the short term (Kim and Duda, 2003) and might be a more effective coping strategy than approach coping (problem-focused coping) (Kaisidis-Rodafinos et al., 1997). Yet, in a long-term perspective, problem-focused coping was positively related to, whereas avoidance was negatively related to, satisfaction, enjoyment and desire to continue (Kim and Duda, 2003). This might imply that avoidance coping can be effective during table tennis competition, but not in the long term. When referring to the Krohne and Hindel's study (1988), it is important to note that they did not measure the perceived control of athletes' emotions during the competitive event. When athletes interpret their anxiety as facilitative, they might assume feeling greater control, while interpreting their anxiety as debilitative might assume they feel less control. This study's results also contradict the Roth and Cohen's study (1986) which suggested different use of coping strategies as a function of perceived control. According to them, approach coping (problem-focused coping) is preferable when stressful situations are perceived as highly controllable, while avoidance coping is more frequently used when there is low perceived control. However, in our study we did not find a significant difference in the effectiveness of these factors.

\section{Table 1}

Significant differences between high and low cognitive anxiety groups in the frequency of the use of emotion focused coping and avoidance

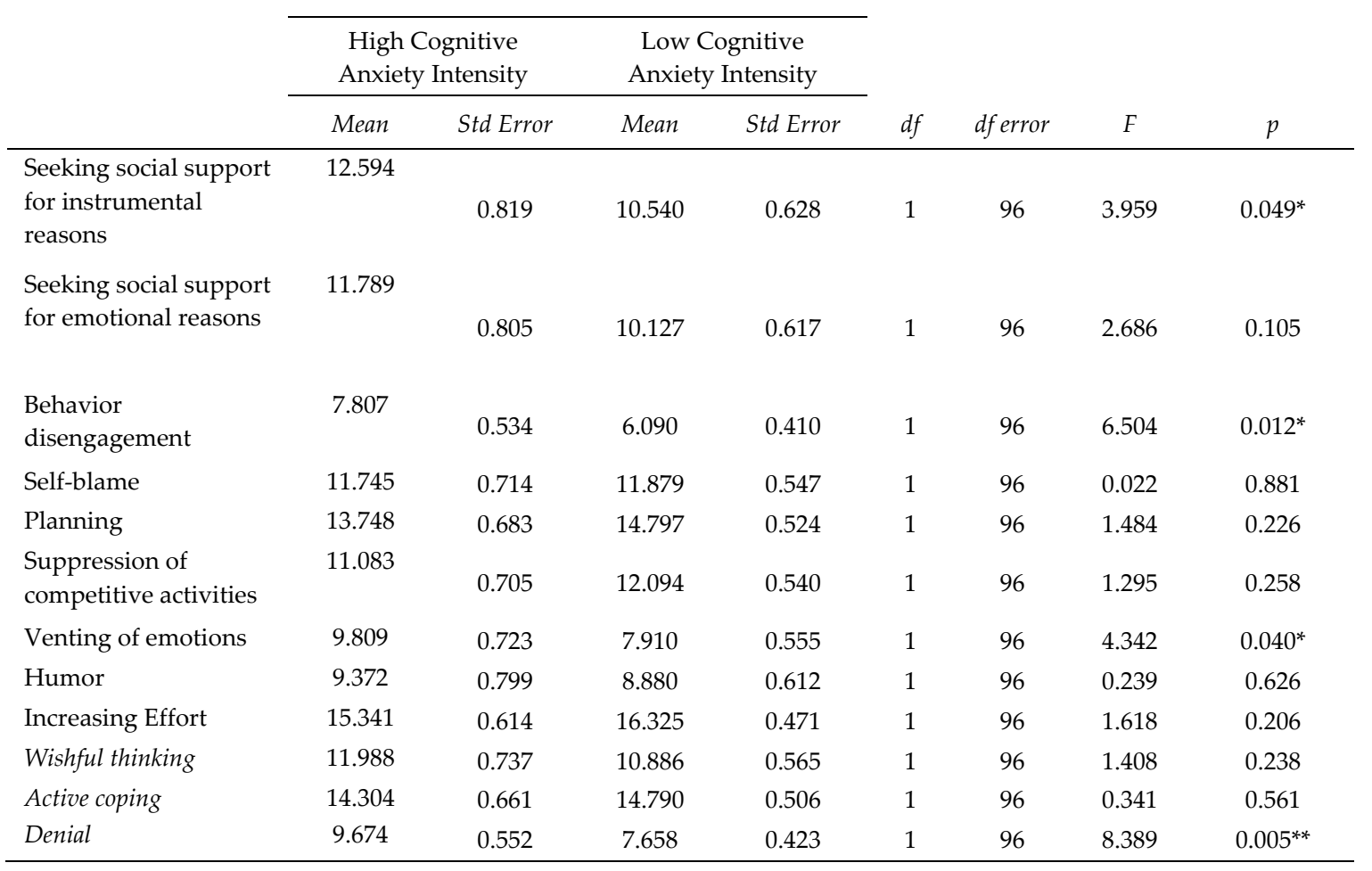

${ }^{* *} p \leq 0.01,{ }^{*} p \leq 0.05$ 

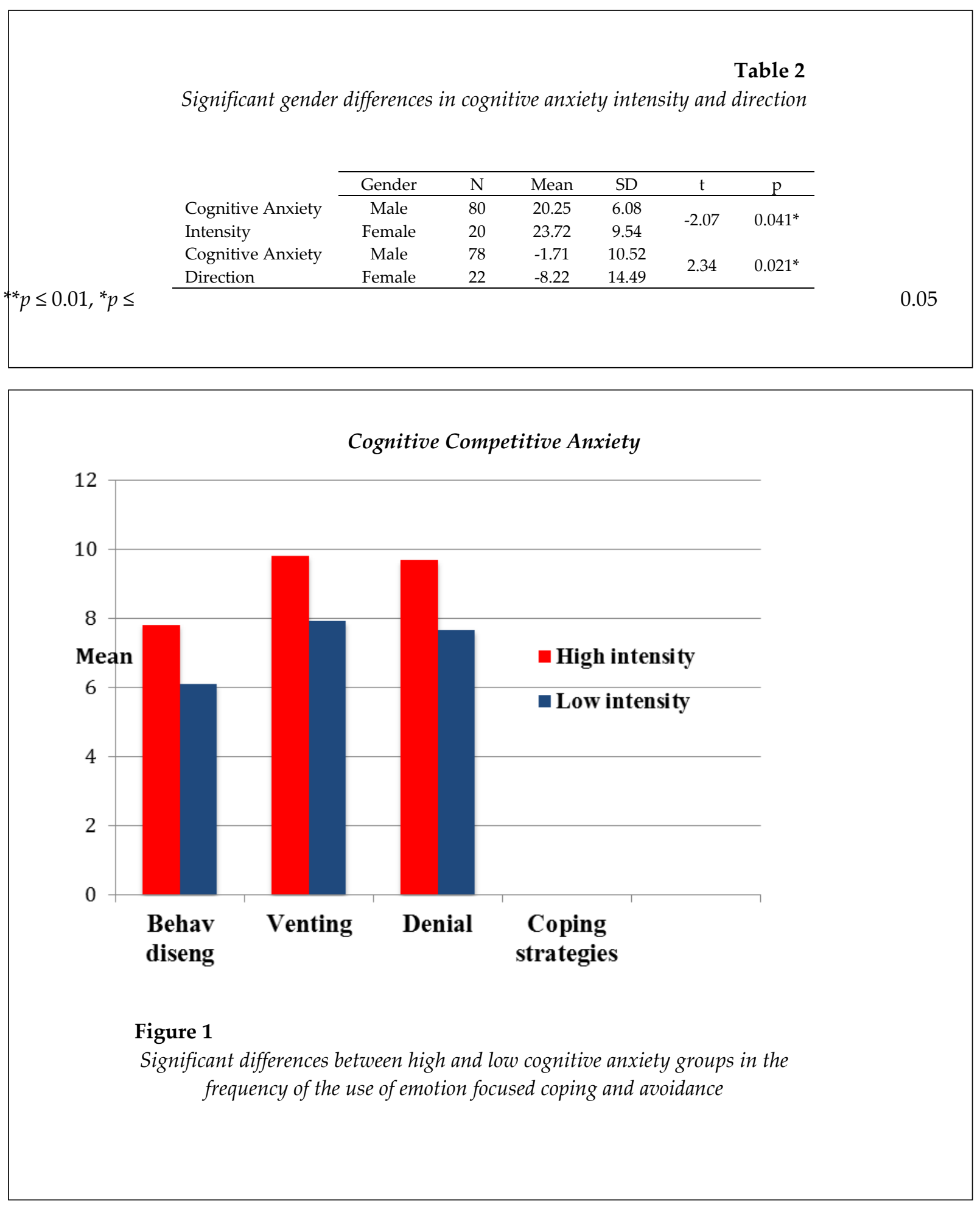

One of the key factors in determining effective coping strategies is how athletes interpret their anxiety (facilitative or debilitative) which in turn can help them cope with stress more effectively and assist in the choice of most appropriate coping strategies (problem-focused, emotion-focused or avoidance coping) in the competition environment.

It is essential to point out that Ntoumous and Biddle's (2000) and Hatzigeorgiadis et al.'s (2007) study participants were British and Greek, respectively. Thus, our results (obtained in US residents) could be different by virtue of cultural differences. Based on previous studies, cultural 
differences could influence coping strategies and perceived controllability (Puente-Diaz and Anshel, 2005).

Another finding of the present study concerned gender differences that did exist in cognitive anxiety intensity and direction. Women rated their cognitive anxiety higher and more negatively compared to male athletes; however, there were significant differences between the number of men $(\mathrm{N}=80)$ and women $(\mathrm{N}=22)$ in the sample, which could distort the results.

This study has several limitations. First, because of the nature of the sample, we can only apply the results to table tennis. Nevertheless, this can also be an opportunity for other sports to compare their results with table tennis. Second, our research did not focus on a particular age group or level per se, therefore, the group of participants is not homogenous in regard to age and sports level of the table tennis players in this study's sample. This could limit some elements for generalization and practical implications. However, it is important to highlight that in the American table tennis tournament system, the age range and sporting level of the participants are typically very wide. Thus, it was very hard to control our sample's homogeneity and ensure a sufficient number of participants in the study.
Future research should repeat this study with a more homogenous sample. Thirdly, the research did not include the factor of winning and losing. It did not take into consideration whether the athletes won or lost when they completed the post-match Modified Cope questionnaire (MCOPE; Crocker and Graham, 1995) to define what coping strategies were most successful in a competitive environment. Furthermore, the findings do not allow us to draw correlational or causal relationships between anxiety intensity, direction and coping strategies.

The results suggest that there is some connection between cognitive competitive anxiety intensity and coping strategies. They indicate that cognitive competitive anxiety intensity might predict what kinds of coping strategies athletes would use during competition.

Based on our findings, coaches and sport psychologists should assess their athletes' level of cognitive anxiety to help them develop effective coping strategies and appropriate stress management techniques. Furthermore, gender differences in competitive anxiety should be considered as well. Confidence and arousal management strategies such as positive self-talk imagery and deep breathing should be taught to athletes to assist them to cope with their competitive anxiety successfully. Additionally, our study also points out that anxiety responses are normal in stressful situations such as competitions and can even be facilitative.

\section{Acknowledgements}

We want to thank all the table tennis players who participated in our study. This project could, literally, not have happened without them. We would like to say a big thank you for Stephanie Buck for her help in researching the literature.

\section{References}

Ansel MH, Si G. Copying styles following acute stress in sport among elite Chinese athletes: a test of trait and transactional copying theories. J Sport Behav, 2008; 31: 3-21

Cox RH, Martens MP, Russell WD. Measuring anxiety in athletics: The revised Competitive State Anxiety Inventory-2. J Sport and Exercise Psy, 2003; 25: 519-533

Crocker PRE, Graham TR. Coping by competitive athletes with performance stress: Gender differences and relationships with affect. Sport Psychol, 1995; 9: 325-338

Gan Q, Anshel MH, Kim JK. Sources and cognitive appraisals of acute stress as predictors of coping style among male and female Chinese athletes. International Journal of Sport and Exercise Psychology, 2009; 7: $68-88$ 
Gillham E, Gillham AD. Identifying athletes' sources of competitive state anxiety. Journal of Sport Behavior, 2014; 37: $1-16$

Hatzigeorgiadis A, Chroni S. Pre-competition anxiety and in-competition coping experienced male swimmers. Int J Sports Sci Coach, 2007; 2: 181-189

Jones G. More than just a game: Research developments and issues in competitive anxiety in sport. British Journal of Psychology, 1995; 86: 449-478

Kaisidis-Rodafinos A, Anshel MA, Porter A. Personal and situational factors that predict coping strategies for acute stress among basketball referees. J Sport Sci, 1997; 15: 427-436

Kashdan TB, Barrios V, Forsyth JP, Steger MF. Experiential avoidance as a generalized psychological vulnerability: Comparisons with coping and emotion regulation strategies. Behav Res Ther, 2006; 44: 1301-1320

Kim MS, Duda JL. The Coping Process: Cognitive appraisal of stress, coping strategies, and coping effectiveness. Sport Psychol, 2003; 17: 406-423

Krohne HW, Hindel C. Trait anxiety, state anxiety, and coping behavior as predictors of athletic performance. Anxiety Res, 1988; 1: 225-234

Mellalieu SD, Hanton S, Fletcher D. A competitive anxiety review: recent directions in sport psychology research. Literature reviews in sport psychology. S. Hanton and SD. Mellalieu (Eds), New York: Nova Science Publishers, 1-45; 2009

Neil R, Mellalieu SD, Hanton S. Psychological skills usage and competitive anxiety response as a function of skill level in rugby union. J Sports Sci Med, 2006; 5: 415-423

Ntoumanis N, Biddle SJH. Relationship of intensity and direction of competitive anxiety with coping strategies. The Sport Psychologist, 2000; 14: 360-371

Patel DR, Omar H, Terry M. Sport-related performance anxiety in young female athletes. J Pediatr Adolesc Gynecol, 2010; 23: 325-335

Puente-Diaz R, Anshel MH. Sources of acute stress, cognitive appraisal, and coping strategies among highly skilled mexican and U.S. competitive tennis players. J Soc Psychol, 2005; 4: 429-446

Radochonski M, Cynarski WJ, Perenc L, Siorek-Maslanka L. Competitive anxiety and coping strategies in youth martial arts and track and field athletes. J Hum Kinet, 2011; 27: 181-190

Roth S, Cohen LJ. Approach avoidance, and coping with stress. American Psychologist, 1986; 41: 813-819

Thomas PR, Murphey SM, Hardy L. Test of performance strategies: Development and preliminary validation of a comprehensive measure of athletes' psychological skills. Journal of Sports Sciences, 1999; 17: 697-711

Wagtsaff CRD, Neil R, Mellalieu SD, Hanton S. Key movements in directional research in competitive anxiety. In Neil R, Mellalieu SD, Hanton S (Eds.) Coping and emotion in sport. London: Taylor \& Francis, 143-166; 2011

Woodman T, Hardy L. Stress and Anxiety. In Singer R, Hausenblas HA, Janelle CM. (Eds.). Handbook of research on sport psychology, New York: Wiley, 290-318; 2001

\section{Corresponding author:}

\section{Dora Kurimay}

Pasaréti út 110/A

Budapest 1026, Hungary

Phone: +36 302205164

E-mail:dora@dorakurimay.com 